\title{
Clinical benefit of glasdegib plus low-dose cytarabine in patients with de novo and secondary acute myeloid leukemia: long-term analysis of a phase II randomized trial
}

\author{
Michael Heuser ${ }^{1}$ (D) B. Douglas Smith ${ }^{2} \cdot$ Walter Fiedler $^{3} \cdot$ Mikkael A. Sekeres $^{4} \cdot$ Pau Montesinos $^{5,6} \cdot$ Brian Leber $^{7}$. \\ Akil Merchant ${ }^{8}$. Cristina Papayannidis ${ }^{9}$ - José A. Pérez-Simón ${ }^{10}$. Caroline J. Hoang ${ }^{11}$. Thomas $\mathrm{O}^{\prime}$ Brien ${ }^{11}$. \\ Weidong Wendy Ma ${ }^{11}$ - Mirjana Zeremski ${ }^{11}$. Ashleigh $\mathrm{O}^{\prime}$ Connell ${ }^{11}$ - Geoffrey Chan ${ }^{11}$ • Jorge E. Cortes ${ }^{12,13}$
}

Received: 10 February 2021 / Accepted: 15 February 2021 / Published online: 19 March 2021

(C) The Author(s) 2021, corrected publication 2021

\begin{abstract}
This analysis from the phase II BRIGHT AML 1003 trial reports the long-term efficacy and safety of glasdegib + low-dose cytarabine (LDAC) in patients with acute myeloid leukemia ineligible for intensive chemotherapy. The multicenter, open-label study randomized (2:1) patients to receive glasdegib + LDAC (de novo, $n=38$; secondary acute myeloid leukemia, $n=40$ ) or LDAC alone (de novo, $n=18$; secondary acute myeloid leukemia, $n=20$ ). At the time of analysis, $90 \%$ of patients had died, with the longest follow-up since randomization 36 months. The combination of glasdegib and LDAC conferred superior overall survival (OS) versus LDAC alone; hazard ratio (HR) 0.495; (95\% confidence interval [CI] 0.325-0.752); $p=0.0004$; median OS was 8.3 versus 4.3 months. Improvement in OS was consistent across cytogenetic risk groups. In a post-hoc subgroup analysis, a survival trend with glasdegib + LDAC was observed in patients with de novo acute myeloid leukemia (HR 0.720; $95 \%$ CI $0.395-$ $1.312 ; p=0.14$; median OS 6.6 vs 4.3 months) and secondary acute myeloid leukemia (HR $0.287 ; 95 \%$ CI $0.151-0.548 ; p<$ 0.0001; median OS 9.1 vs 4.1 months). The incidence of adverse events in the glasdegib + LDAC arm decreased after 90 days' therapy: $83.7 \%$ versus $98.7 \%$ during the first 90 days. Glasdegib + LDAC versus LDAC alone continued to demonstrate superior OS in patients with acute myeloid leukemia; the clinical benefit with glasdegib + LDAC was particularly prominent in patients with secondary acute myeloid leukemia. ClinicalTrials.gov identifier: NCT01546038.
\end{abstract}

Keywords Acute myeloid leukemia $\cdot$ Clinical trial $\cdot$ Glasdegib $\cdot$ Secondary acute myeloid leukemia

Michael Heuser

Heuser.Michael@mh-hannover.de

1 Department of Hematology, Hemostasis, Oncology and Stem Cell Transplantation, Hannover Medical School, Carl-Neuberg-Str. 1, 30625 Hannover, Germany

2 Johns Hopkins Sidney Kimmel Comprehensive Cancer Center, Baltimore, MD, USA

3 Department of Hematology and Oncology, University Hospital Hamburg-Eppendorf, Hamburg, Germany

4 Division of Hematology, Sylvester Comprehensive Cancer Center, University of Miami, FL Miami, USA

5 Hospital Universitari i Politècnic La Fe, Valencia, Spain

6 CIBERONC, Instituto Carlos III, Madrid, Spain
7 Juravinski Hospital at Hamilton Health Sciences, Hamilton, ON, Canada

8 Samuel Oschin Comprehensive Cancer Institute, Cedars-Sinai Medical Center, Los Angeles, CA, USA

9 IRCCS Azienda Ospedaliero-Universitaria di Bologna, Bologna, Italy

10 Hospital Universitario Virgen del Rocío, Instituto de Biomedicina (IbiS)/CSIC/CIBERONC), Universidad de Sevilla, Seville, Spain

11 Pfizer Inc, New York, NY, USA

12 University of Texas MD Anderson Cancer Center, Houston, TX, USA

13 Present address: Georgia Cancer Center, Augusta, GA, USA 


\section{Introduction}

Acute myeloid leukemia (AML) is the most common type of leukemia in adults [1]. Secondary AML is associated with lower response rates and decreased overall survival (OS) with standard chemotherapy compared with de novo AML [2-6]. Although most cases arise de novo, AML can evolve from an antecedent myeloid disease or as a late complication from chemotherapy or ionizing radiation. Secondary AML accounts for approximately $25 \%$ of all AML cases (18-20\% from a previous myeloid disease; $6-8 \%$ related to therapy) and occurs more frequently with increasing age $[5,7]$. The incidence of secondary AML is highest between the ages of 70 and 79 years $[7,8]$. Few randomized trials have been conducted that report data by diagnosis of de novo and secondary AML.

Induction with $7+3$ chemotherapy has long remained the conventional treatment for patients with AML. In older adults receiving $7+3$ regimens, induction mortality rates are higher, and response rates are lower than in younger individuals $[6$, 9]. Outcomes for older adults with secondary AML are particularly poor with $7+3$ regimens. A recent phase III clinical trial demonstrated that CPX-351 (liposomal cytarabine and daunorubicin) significantly improved OS in patients aged $\geq$ 60 years with secondary AML versus standard $7+3$ induction chemotherapy (9.56 vs 5.95 months, $p=0.0005$ ) [10]. However, older patients with AML are often ineligible for such intensive chemotherapy because of comorbidities, performance status, or disease-related chemoresistance; therefore, they are treated with less-aggressive therapies including low-dose cytarabine (LDAC) and hypomethylating agents (HMAs; decitabine or azacitidine) [11, 12]. Studies with LDAC, decitabine, and azacitidine in older patients with AML have demonstrated median OS rates of 5 months, 7.7 months, and 10.4 months, respectively, indicating the need for novel therapeutic strategies to improve survival [13-15]. Several novel agents have recently been approved in the USA for the treatment of patients ineligible for intensive chemotherapy (e.g., glasdegib and venetoclax) $[16,17]$.

Glasdegib is a potent, selective, oral inhibitor of the Hedgehog signaling pathway. In a phase II randomized study that included patients with newly diagnosed AML or high-risk myelodysplastic syndromes (MDS) who were ineligible for intensive chemotherapy, the addition of glasdegib to LDAC demonstrated superior OS versus LDAC alone [18]. Based on the primary analysis of BRIGHT AML 1003, glasdegib was approved in the USA and Europe in combination with LDAC for the treatment of patients with newly diagnosed AML who are unable to receive intensive chemotherapy as a result of comorbidities or older age ( $\geq 75$ years) $[19,20]$.

Here we report the efficacy and safety of glasdegib + LDAC in patients with AML after approximately 20 additional months of follow-up from the primary completion date.
Exploratory analyses assessing the clinical benefit and safety of glasdegib + LDAC in AML subgroups based on de novo or secondary disease status are also reported.

\section{Methods}

\section{Study design and patients}

BRIGHT AML 1003 was an open-label, randomized, multicenter, phase II study (ClinicalTrials.gov identifier: NCT01546038) for which the methods have previously been published [18]. Briefly, BRIGHT AML 1003 enrolled adult patients aged $\geq 55$ years with newly diagnosed, previously untreated AML or high-risk MDS (World Health Organization 2008 classification), who were ineligible for intensive chemotherapy, defined as meeting $\geq 1$ of the following criteria: $\geq 75$ years old; severe cardiac disease; baseline Eastern Cooperative Oncology Group performance status $($ ECOG PS $)=2$; or baseline serum creatinine $>1.3 \mathrm{mg} / \mathrm{dL}$ [19]. Glasdegib $100 \mathrm{mg}$ was administered orally, once daily, on a continuous basis. LDAC $20 \mathrm{mg}$ was administered subcutaneously, twice daily for 10 days, every 28 days. Treatments continued until disease progression, unacceptable toxicity, or patient refusal. This long-term analysis assessed efficacy and safety in patients with AML only.

The study was conducted in accordance with the Declaration of Helsinki. All patients provided written informed consent before study procedures began, and the protocol was approved by institutional review boards at each study site.

\section{Efficacy and safety assessments}

The study's primary endpoint was OS. The secondary endpoint of response to treatment was assessed based on the International Working Group response criteria for AML [21].

Transfusion rates and recovery of the three blood cell lineages, all at two thresholds, were also measured: absolute neutrophil count $(\geq 1000 / \mu \mathrm{L}$ or $500 / \mu \mathrm{L})$; hemoglobin $(\geq 10$ $\mathrm{g} / \mathrm{dL}$ or $9 \mathrm{~g} / \mathrm{dL}$ ); and platelets $(\geq 100,000 / \mu \mathrm{L}$ or $50,000 / \mu \mathrm{L})$. Time to recovery and treatment cycle analyses included only remaining patients at risk in that cycle. Transfusion independence was defined as $\geq 8$ weeks without transfusions.

Safety assessments included adverse events (AEs), classified and graded based on the National Cancer Institute Common Terminology Criteria for Adverse Events v4.0, laboratory evaluations, vital signs, physical examinations, and 12-lead electrocardiograms.

A post-hoc subgroup analysis was also performed in patients categorized by diagnosis as determined by the investigator: de novo AML or secondary AML. Secondary AML was defined as AML evolving from MDS or other antecedent 
hematologic disease, or AML after previous cytotoxic therapy or radiation.

\section{Biomarker analysis}

Biomarker assessments included determination of baseline mutational status of the following genes: CEBPA, DNMT3A, FLT3, IDH1, IDH2, KIT, KRAS, NPM1, NRAS, RUNX1, TET2, and WT1.

\section{Statistical analyses}

After discontinuation of study treatment, patients were followed until death or for 4 years from the first dose. OS was estimated using the Kaplan-Meier method, and 95\% confidence intervals (CIs) of median OS were calculated using the Brookmeyer and Crowley method. For biomarker analyses, a Cox proportional hazard regression model was used to estimate the hazard ratio (HR) and $95 \%$ CI of OS using the non-mutated gene or the LDAC alone arm as the reference group. Exposure-adjusted transfusion rates were calculated as the sum of on-study transfusions/total time on treatment for all patients in the treatment arm. Other efficacy endpoints were summarized descriptively. Safety data were also summarized descriptively and included all randomized patients who received at least one dose of any of the study medications.

\section{Results}

\section{Disposition, demography, and baseline characteristics}

One hundred and sixteen patients with AML were randomized (2:1) to treatment with glasdegib + LDAC (de novo, $n=38$; secondary AML, $n=40$ ) or LDAC alone (de novo, $n=18$; secondary AML, $n=20$ ); among them, 75 (de novo, $n=38$; secondary AML, $n=37$ ) and 36 (de novo, $n=17$; secondary AML, $n=19$ ) patients received study treatments, respectively (Online Resource, Fig. S1). At the time of data cut-off (11 October 2018), three patients in the glasdegib + LDAC arm (de novo, $n=1$; secondary AML, $n=2$ ) had received $\geq 172$ weeks of treatment and were still receiving therapy. The most common reason for treatment discontinuation among all patient cohorts was insufficient clinical response.

Baseline demographics and disease characteristics were generally similar across the two treatment arms (Table 1). At study entry, the median age was 77 years (range, 64-92) in the glasdegib + LDAC arm and 76 years (range, 58-83) in the LDAC arm; $51.3 \%$ and $52.6 \%$ of patients had a diagnosis of secondary AML, respectively. In the overall population (de novo + secondary AML), the median duration of treatment was 83.0 days (range, 3-1492) and 40.5 days (range, 6-239) in the glasdegib + LDAC and LDAC alone arms, respectively;
13/75 patients $(17.3 \%)$ were treated with glasdegib + LDAC for at least 1 year (Online Resource, Table S1). The median duration of treatment for glasdegib + LDAC and LDAC alone, respectively, was 69.5 days (range, 5-1206) and 47.0 days (range, 10-239) in the de novo AML subgroup and 101.0 days (range, 3-1492) and 39.0 days (range, 6-149) in the secondary AML subgroup.

Among patients who received treatment, 34/75 (45.3\%) in the glasdegib + LDAC arm and 11/36 (30.6\%) in the LDAC alone arm were reported to have received subsequent systemic therapies after discontinuation of the study treatment. The most frequent subsequent therapy was HMAs in 52.9\% (18/ 34 ) and $36.4 \%$ (4/11) of patients in the glasdegib + LDAC and LDAC alone arm, respectively.

In the overall AML population, at the time of analysis, $90 \%$ of patients had died, with the longest follow-up time since randomization 36 months.

\section{Efficacy}

In the overall AML population, treatment with glasdegib + LDAC resulted in superior OS versus LDAC alone (HR 0.495 ; 95\% CI 0.325-0.752; $p=0.0004)$; median OS was 8.3 (95\% CI 4.7-12.2) versus 4.3 (95\% CI 1.9-5.7) months (Fig. 1a and Table 2). The respective survival probability was $39.4 \%$ (95\% CI 28.3-50.3) and 8.4\% (95\% CI 2.2-20.1) at 1 year, and $19.0 \%$ (95\% CI 11.0-28.7) and 2.8\% (95\% CI $0.2-$ 12.4) at 2 years. The improvement in survival with glasdegib + LDAC was consistent across most groups stratified by cytogenetic risk (Table 2).

In the de novo AML subgroup, median OS was $6.6(95 \%$ CI 3.7-12.4) months with glasdegib + LDAC, and $4.3(95 \%$ CI 1.3-10.7) months with LDAC alone (HR 0.720; $95 \%$ CI $0.395-1.312 ; p=0.1398$ ) (Fig. $1 \mathrm{~b}$ and Table 2). The respective survival probability was $40.4 \%$ (95\% CI 24.3-55.9) and $17.6 \%$ (95\% CI 4.3-38.3) at 1 year, and $11.5 \%$ (95\% CI 3.7$24.4)$ and $5.9 \%(95 \%$ CI $0.4-23.5)$ at 2 years. The survival benefit of glasdegib + LDAC versus LDAC alone was more pronounced among patients in the secondary AML subgroup (HR 0.287; 95\% CI 0.151-0.548; $p<0.0001$ ); median OS was 9.1 (95\% CI 4.4-16.5) months and 4.1 (95\% CI 1.56.4) months, respectively (Fig. 1c). The survival probabilities at 1 and 2 years, respectively, were $38.5 \%$ (95\% CI 23.6-53.3) and $25.7 \%$ (95\% CI 13.3-39.9) with glasdegib + LDAC, and $0 \%$ with LDAC alone (all patients died within the first year).

Among patients in the overall population who received prior therapy with HMAs (glasdegib + LDAC, $n=11$; LDAC alone, $n=6$ ), the median OS was 7.1 (95\% CI 2.214.7) months with glasdegib + LDAC and 5.1 (95\% CI $0.5-$ 7.2) months with LDAC alone (HR 0.438 ; $95 \%$ CI 0.138 $1.391 ; p=0.0754)$. Of the patients in the overall population without prior HMA therapy (glasdegib + LDAC, $n=67$; LDAC alone, $n=32)$, the median OS was 8.8 (95\% CI $4.7-$ 
Table 1 Demography and baseline characteristics by diagnosis of AML

\begin{tabular}{|c|c|c|c|c|c|c|}
\hline \multirow[t]{2}{*}{ Characteristic } & \multicolumn{2}{|l|}{ Overall population } & \multicolumn{2}{|l|}{ de novo AML } & \multicolumn{2}{|l|}{ Secondary AML } \\
\hline & $\begin{array}{l}\text { Glasdegib + LDAC } \\
n=78\end{array}$ & $\begin{array}{l}\text { LDAC alone } \\
n=38\end{array}$ & $\begin{array}{l}\text { Glasdegib + LDAC } \\
n=38\end{array}$ & $\begin{array}{l}\text { LDAC alone } \\
n=18\end{array}$ & $\begin{array}{l}\text { Glasdegib + LDAC } \\
n=40\end{array}$ & $\begin{array}{l}\text { LDAC alone } \\
n=20\end{array}$ \\
\hline \multicolumn{7}{|l|}{ Sex, $n(\%)$} \\
\hline Female & $19(24.4)$ & $15(39.5)$ & $10(26.3)$ & $8(44.4)$ & $9(22.5)$ & $7(35.0)$ \\
\hline Male & $59(75.6)$ & $23(60.5)$ & $28(73.7)$ & $10(55.6)$ & $31(77.5)$ & $13(65.0)$ \\
\hline \multicolumn{7}{|l|}{ Age, years, $n(\%)$} \\
\hline Mean (SD) & $76.4(6.0)$ & $74.8(4.9)$ & $76.6(5.8)$ & $74.6(5.1)$ & $76.2(6.3)$ & $74.9(4.9)$ \\
\hline Median (range) & $77.0(64-92)$ & $76.0(58-83)$ & $77.0(64-87)$ & $75.5(58-80)$ & $76.0(65-92)$ & $76.0(65-83)$ \\
\hline \multicolumn{7}{|l|}{ Secondary AML, $n(\%)$} \\
\hline Prior hematologic disease & 34 (43.6) & $19(50.0)$ & - & - & $34(85.0)$ & $19(95.0)$ \\
\hline MDS & $29(37.2)$ & $15(39.5)$ & - & - & $29(72.5)$ & $15(75.0)$ \\
\hline Other & $5(6.4)$ & $4(10.5)$ & - & - & $5(12.5)$ & $4(20.0)$ \\
\hline Chemotherapy/ radiotherapy & $6(7.7)$ & $1(2.6)$ & - & - & $6(15.0)$ & $1(5.0)$ \\
\hline \multicolumn{7}{|l|}{ Prior therapy with MDS drug, $n(\%)$} \\
\hline Azacitidine & $10(12.8)$ & $5(13.2)$ & - & - & $10(25.0)$ & $5(25.0)$ \\
\hline Decitabine & $1(1.3)$ & $1(2.6)$ & - & - & $1(2.5)$ & $1(5.0)$ \\
\hline Duration since diagnosis, median, months & 0.6 & 0.5 & 0.5 & 0.5 & 0.6 & 0.6 \\
\hline \multicolumn{7}{|c|}{ First-line AML non-intensive population criteria, $n(\%)$} \\
\hline Age $\geq 75$ years & $48(61.5)$ & $23(60.5)$ & $25(65.8)$ & $12(66.7)$ & $23(57.5)$ & $11(55.0)$ \\
\hline ECOG PS $=2$ & $41(52.6)$ & $18(47.4)$ & $18(47.4)$ & $8(44.4)$ & $23(57.5)$ & $10(50.0)$ \\
\hline $\mathrm{sCr}>1.3 \mathrm{mg} / \mathrm{dL}$ & $15(19.2)$ & $5(13.2)$ & 7 (18.4) & $4(22.2)$ & $8(20.0)$ & $1(5.0)$ \\
\hline Severe cardiac disease & $52(66.7)$ & $20(52.6)$ & $29(76.3)$ & $9(50.0)$ & $23(57.5)$ & $11(55.0)$ \\
\hline \multicolumn{7}{|l|}{ Cytogenetic risk, $n(\%)^{\mathrm{a}}$} \\
\hline Good/intermediate & $53(67.9)$ & $22(57.9)$ & $25(65.8)$ & $14(77.8)$ & $28(70.0)$ & $8(40.0)$ \\
\hline Poor & $25(32.1)$ & $16(42.1)$ & $13(34.2)$ & $4(22.2)$ & $12(30.0)$ & $12(60.0)$ \\
\hline \multicolumn{7}{|l|}{ ELN risk stratification for AML, $n(\%)[22]$} \\
\hline Favorable & $5(6.4)$ & $3(7.9)$ & $3(7.9)$ & $2(11.1)$ & $2(5.0)$ & $1(5.0)$ \\
\hline Intermediate I & $27(34.6)$ & $11(28.9)$ & $13(34.2)$ & $5(27.8)$ & $14(35.0)$ & $6(30.0)$ \\
\hline Intermediate II & $21(26.9)$ & $8(21.1)$ & $9(23.7)$ & $7(38.9)$ & $12(30.0)$ & $1(5.0)$ \\
\hline Adverse & $25(32.1)$ & $16(42.1)$ & $13(34.2)$ & $4(22.2)$ & $12(30.0)$ & $12(60.0)$ \\
\hline \multicolumn{7}{|l|}{ Mutations, $n(\%)^{\mathrm{b}}$} \\
\hline FLT3 & $5(6.4)$ & 0 & $3(7.9)$ & 0 & $2(5.0)$ & 0 \\
\hline$I D H 1$ or $I D H 2$ & $19(24.3)$ & $6(15.8)$ & $14(29.2)$ & $2(11.1)$ & $5(12.5)$ & $4(20.0)$ \\
\hline \multirow{2}{*}{\multicolumn{7}{|c|}{ White blood cell count $\left(10^{3} / \mathrm{mm}^{3}\right)$}} \\
\hline & & & & & & \\
\hline Median (range) & $2.7(0.4-5850.0)$ & $3.8(1.2-1370.0)$ & $2.7(0.4-28.0)$ & $4.0(1.6-27.9)$ & $2.7(0.5-5850.0)$ & $3.7(1.2-1370.0)$ \\
\hline \multicolumn{7}{|l|}{ Hemoglobin $(\mathrm{g} / \mathrm{dL})$} \\
\hline Median (range) & $8.7(6.9-13.8)$ & $9.0(6.9-13.4)$ & $8.8(6.9-13.8)$ & $9.2(7.4-12.4)$ & $8.7(7.3-12.2)$ & $8.9(6.9-13.4)$ \\
\hline \multicolumn{7}{|l|}{ Platelet count $\left(10^{3} / \mathrm{mm}^{3}\right)$} \\
\hline Median (range) & $42.0(7.0-35,000.0)$ & $26.5(3.0-23,000.0)$ & $55.0(10.0-258.0)$ & $34.0(11.0-199.0)$ & $30.0(7.0-35,000.0)$ & $22.0(3.0-23,000.0)$ \\
\hline \multicolumn{7}{|l|}{ Bone marrow blasts, $\%$} \\
\hline Median (range) & $41.0(16.0-99.0)$ & $46.0(13.0-95.0)$ & $47.5(20.8-99.0)$ & $50.5(20.0-87.0)$ & $38.0(16.0-95.0)$ & $43.0(13.0-95.0)$ \\
\hline
\end{tabular}

$A M L$, acute myeloid leukemia; ECOG PS, Eastern Cooperative Oncology Group performance status; ELN, European LeukemiaNet; $L D A C$, low-dose cytarabine; $M D S$, myelodysplastic syndromes; $s C r$, serum creatinine; $S D$, standard deviation

${ }^{a}$ For AML, good/intermediate cytogenetic risk = favorable, Intermediate I and Intermediate II risk groups; poor cytogenetic risk = adverse risk group

${ }^{\mathrm{b}}$ Baseline gene mutations were determined in 58/78 glasdegib/LDAC patients (de novo AML, $n=30$; secondary AML, $n=28$ ) and 25/38 LDAC alone patients (de novo AML, $n=11$; secondary AML, $n=14$ )

12.4) months with glasdegib + LDAC and $4.1(95 \%$ CI 1.8 6.4) months with LDAC alone (HR 0.500; 95\% CI $0.317-$ $0.789 ; p=0.0012)$; among patients with secondary AML (glasdegib + LDAC, $n=29$; LDAC alone, $n=14$ ), the median OS was 9.9 (95\% CI 6.5-19.6) months with glasdegib + LDAC and 2.9 (95\% CI 1.0-6.4) months with LDAC alone. For patients who did not receive subsequent HMAs in the overall population, the median OS was 6.5 (95\% CI 3.5-8.8) months with glasdegib + LDAC and 3.5 (95\% CI 1.8-4.9) months with LDAC alone (Fig. 1d); among patients with secondary AML, the median OS was 7.4 (95\% CI 3.1-9.9) months with glasdegib + LDAC and 2.9 (95\% CI 1.5-5.3) months with LDAC alone.
To further define the survival benefit of glasdegib + LDAC, a subgroup analysis was performed by baseline characteristics. The improvement in OS with glasdegib + LDAC was consistent across most subgroups in the overall population (Fig. 2), and by the diagnosis of de novo and secondary AML (Online Resource, Fig. S2 and Online Resource, Fig. S3). Consistent with a benefit in secondary AML, patients with less than $30 \%$ blasts at diagnosis and patients who developed AML from a prior hematologic disease derived a survival benefit from glasdegib + LDAC.

In the overall AML population, 15 patients $(19.2 \%)$ in the glasdegib + LDAC arm and one patient $(2.6 \%)$ in the LDAC alone arm achieved complete remission (CR). The median 
duration of CR was 302 days in the glasdegib + LDAC arm and 91 days in the LDAC alone arm. The rates of CR were similar for patients with de novo $(18.4 \%)$ and secondary (20.0\%) AML in the glasdegib + LDAC arm; however, duration of remission was longer in patients with secondary AML (532 days) versus de novo AML (175 days) (Table 3). Among patients in the overall population who received prior therapy with HMAs, 9.1\% $(n=1 / 11)$ of patients in the glasdegib + LDAC arm and no patients $(n=0 / 6)$ in the LDAC alone arm achieved CR; the median duration of CR was 64 days in the glasdegib + LDAC arm. Of the patients in the overall population without prior HMA therapy, $20.9 \%(n=14 / 67)$ in the glasdegib + LDAC arm and $3.1 \%(n=1 / 32)$ in the LDAC alone arm achieved $\mathrm{CR}$; among patients with secondary AML, 24.1\% $(n=7 / 29)$ in the glasdegib + LDAC arm and no patients $(n=0 / 14)$ in the LDAC alone arm achieved CR. In the overall population, the median duration of CR was 379.5 (95\% CI 1-1262) days with glasdegib + LDAC and 91 days with LDAC alone; for patients with secondary AML receiving glasdegib +
LDAC, the median duration of CR was 574.0 (95\% CI 302-1262) days.

zIn the overall AML population, more patients achieved durable ( $\geq 2$ consecutive assessments) recovery of absolute neutrophil count, hemoglobin, and platelets in the glasdegib + LDAC arm than in the LDAC alone arm (Table 4 and Online Resource, Fig. S4). The median time to recovery with glasdegib + LDAC versus LDAC alone was longer for absolute neutrophil count ( $\geq 1000 / \mu \mathrm{L}, 27$ vs 13 days; $\geq 500 / \mu \mathrm{L}, 16$ vs 11 days), shorter for hemoglobin $(\geq 10 \mathrm{~g} / \mathrm{dL}, 22$ vs 33 days; $\geq 9$ $\mathrm{g} / \mathrm{dL}, 14$ vs 22 days), and similar for platelets $(\geq$ $100,000 / \mu \mathrm{L}, 30$ vs 26 days; $\geq 50,000 / \mu \mathrm{L}, 26$ vs 24 days). Patients in the glasdegib + LDAC arm had fewer transfusions than those receiving LDAC alone; the difference was more significant in favor of patients receiving glasdegib + LDAC when adjusted for duration of treatment (Table 4). In the overall population, transfusion independence was achieved by $29.3 \%$ of patients receiving glasdegib + LDAC and $5.6 \%$ of patients

Table 2 Overall survival in patients with AML

\begin{tabular}{|c|c|c|c|c|c|c|}
\hline & \multicolumn{2}{|c|}{ Overall population } & \multicolumn{2}{|l|}{ de novo AML } & \multicolumn{2}{|c|}{ Secondary AML } \\
\hline & $\begin{array}{l}\text { Glasdegib + } \\
\text { LDAC }\end{array}$ & LDAC alone & $\begin{array}{l}\text { Glasdegib + } \\
\text { LDAC }\end{array}$ & LDAC alone & $\begin{array}{l}\text { Glasdegib + } \\
\text { LDAC }\end{array}$ & LDAC alone \\
\hline All patients & $n=78$ & $n=38$ & $n=38$ & $n=18$ & $n=40$ & $n=\mathbf{2 0}$ \\
\hline Median OS, months (95\% CI) & $8.3(4.7-12.2)$ & $4.3(1.9-5.7)$ & $6.6(3.7-12.4)$ & $4.3(1.3-10.7)$ & $9.1(4.4-16.5)$ & $4.1(1.5-6.4)$ \\
\hline $\mathrm{HR}(95 \% \mathrm{CI})$ & \multicolumn{2}{|c|}{$0.495(0.325-0.752)$} & \multicolumn{2}{|c|}{$0.720(0.395-1.312)$} & \multicolumn{2}{|c|}{$0.287(0.151-0.548)$} \\
\hline$p$ value & \multicolumn{2}{|l|}{0.0004} & \multicolumn{2}{|l|}{0.1398} & \multicolumn{2}{|l|}{$<0.0001$} \\
\hline \multicolumn{7}{|l|}{ Deaths, $n(\%)$} \\
\hline Total & $69(88.5)$ & $35(92.1)$ & $33(86.8)$ & $16(88.9)$ & $36(90.0)$ & $19(95.0)$ \\
\hline Cause of death: disease progression & $59(75.6)$ & $29(76.3)$ & $28(73.7)$ & $12(66.7)$ & $31(77.5)$ & $17(85.0)$ \\
\hline $\begin{array}{l}\text { Good/intermediate cytogenetic risk, } n \\
(\%)^{\mathrm{a}}\end{array}$ & $53(67.9)$ & $22(57.9)$ & 25 (65.8) & $14(77.8)$ & $28(70.0)$ & $8(40.0)$ \\
\hline Median OS, months (95\% CI) & $12.2(6.9-16.5)$ & $5.3(3.5-8.7)$ & $12.2(3.7-14.9)$ & $4.3(1.3-10.7)$ & $11.1(6.5-24.4)$ & $6.9(4.1-8.1)$ \\
\hline $\mathrm{HR}(95 \% \mathrm{CI})$ & \multicolumn{2}{|c|}{$0.510(0.294-0.886)$} & \multicolumn{2}{|c|}{$0.603(0.295-1.233)$} & \multicolumn{2}{|c|}{$0.350(0.135-0.907)$} \\
\hline$p$ value & \multicolumn{2}{|l|}{0.0074} & \multicolumn{2}{|l|}{0.0792} & \multicolumn{2}{|l|}{0.0121} \\
\hline \multicolumn{7}{|l|}{ Deaths, $n(\%)$} \\
\hline Total & $45(84.9)$ & $19(86.4)$ & $21(84.0)$ & $12(85.7)$ & $24(85.7)$ & $7(87.5)$ \\
\hline Cause of death: disease progression & $37(69.8)$ & $15(68.2)$ & $16(64.0)$ & $9(64.3)$ & $21(75.0)$ & $6(75.0)$ \\
\hline Poor cytogenetic risk, $n(\%)^{\mathrm{b}}$ & $25(32.1)$ & $16(42.1)$ & $13(34.2)$ & $4(22.2)$ & $12(30.0)$ & $12(60.0)$ \\
\hline Median OS, months (95\% CI) & $4.4(2.6-7.4)$ & $2.1(1.0-4.9)$ & $4.1(1.9-8.8)$ & $4.4(1.1-12.9)$ & $5.7(0.2-9.1)$ & $1.8(0.5-4.9)$ \\
\hline $\mathrm{HR}(95 \% \mathrm{CI})$ & \multicolumn{2}{|c|}{$0.514(0.264-1.000)$} & \multicolumn{2}{|c|}{$1.077(0.337-3.441)$} & \multicolumn{2}{|c|}{$0.301(0.109-0.829)$} \\
\hline$p$ value & \multicolumn{2}{|l|}{0.0229} & \multicolumn{2}{|l|}{0.5495} & \multicolumn{2}{|l|}{0.0073} \\
\hline \multicolumn{7}{|l|}{ Deaths, $n(\%)$} \\
\hline Total & $24(96.0)$ & $16(100.0)$ & $12(92.3)$ & $4(100.0)$ & $12(100.0)$ & $12(100.0)$ \\
\hline Cause of death: disease progression & $22(88.0)$ & $14(87.5)$ & $12(92.3)$ & $3(75.0)$ & $10(83.3)$ & $11(91.7)$ \\
\hline
\end{tabular}

$A M L$, acute myeloid leukemia; $C I$, confidence interval; $H R$, hazard ratio; $L D A C$, low-dose cytarabine; $O S$, overall survival

${ }^{\text {a }}$ Favorable, Intermediate I and Intermediate II risk groups

${ }^{\mathrm{b}}$ Adverse risk group 
Fig. 1 Kaplan-Meier plots of overall survival in the a overall population, b de novo AML subgroup, c secondary AML subgroup, and d overall population censoring for patients receiving follow-up HMAs.

GLAS, glasdegib; mo, months; mOS, median overall survival

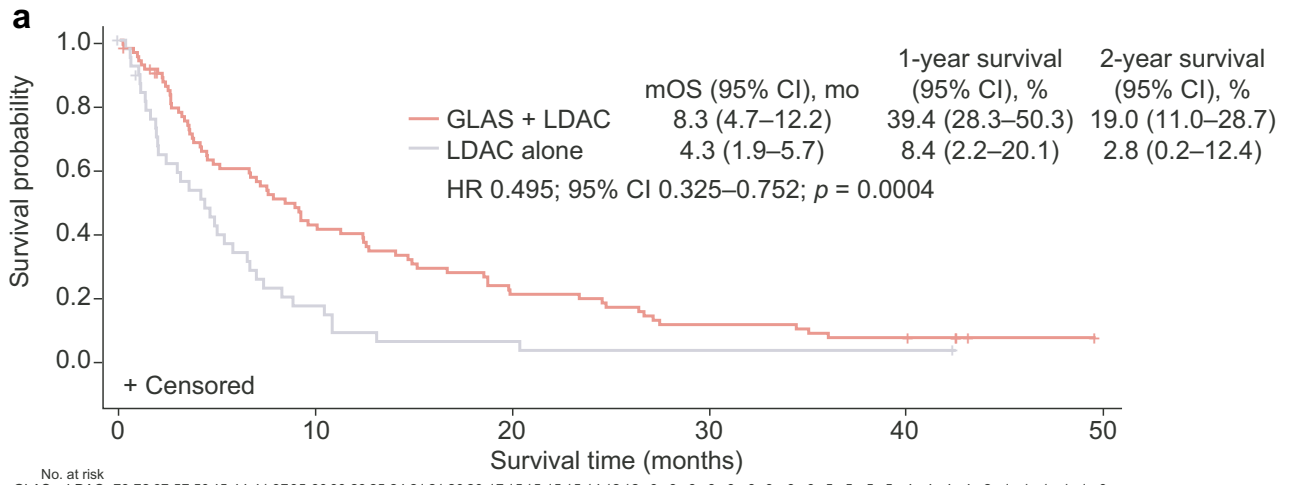

\begin{tabular}{l} 
No. at risk \\
GLAS+LDAC 78726757504544413735303029252421212020171515151514212 \\
LDAC alone 3832232191412998 \\
\hline
\end{tabular}

b

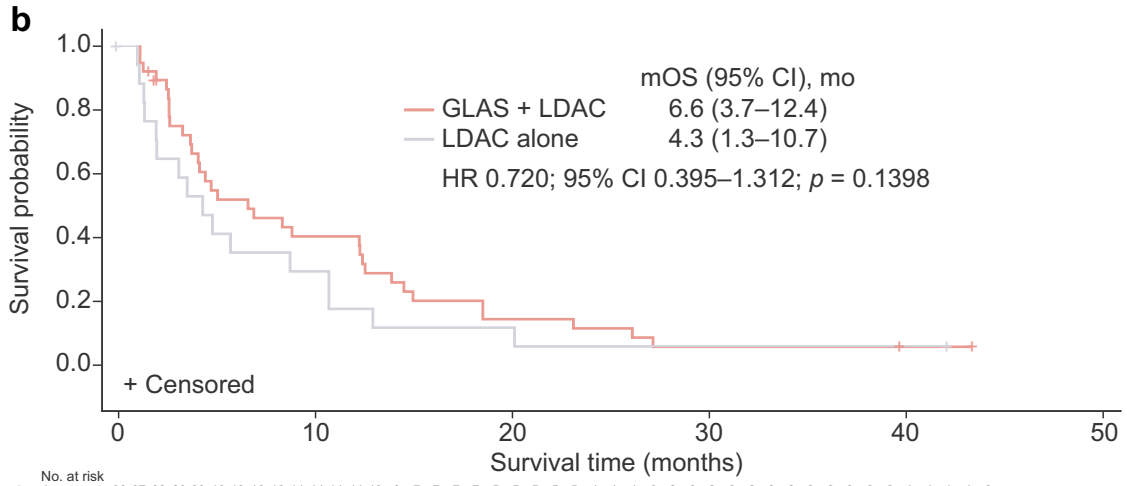

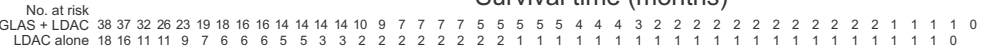

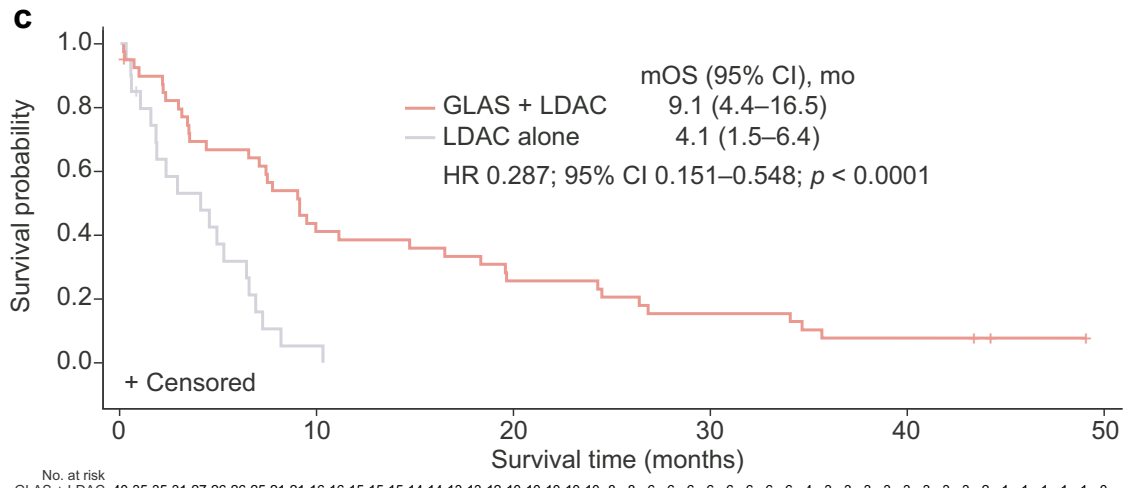

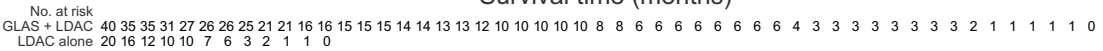

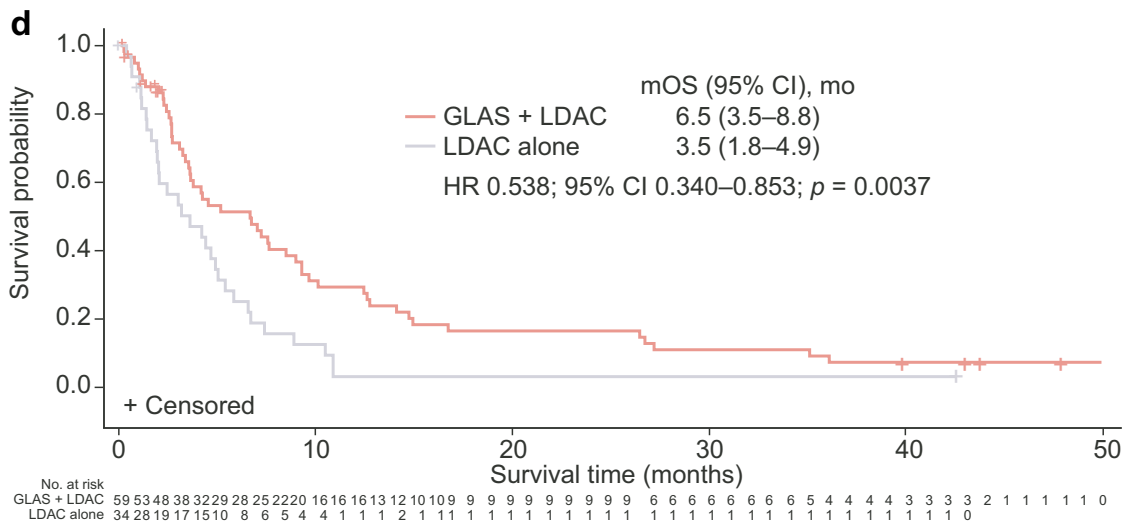

receiving LDAC alone; the median duration of independence was 212 days (range, 56-1054) and 144 days (range, 141-147), respectively. Bone marrow recovery and transfusion independence occurred at similar rates in the de novo and secondary AML subgroups (Online Resource, Table S2). 


\section{Safety}

To account for imbalances in treatment duration between the glasdegib + LDAC ( $\leq 90$ days, $n=75 ;>90$ days, $n=43)$ and LDAC alone ( $\leq 90$ days, $n=36 ;>90$ days, $n=14$ ) arms, AEs are presented separately for the first 90 days. For patients completing $>90$ days of treatment, the median duration of treatment was 262.5 days (range, 97-1492) and 122.5 days (range, 94-239) in the glasdegib + LDAC and LDAC alone arms, respectively. After 90 days, the mean relative dose intensity for glasdegib was $89.4 \%$ for the glasdegib + LDAC arm, and the mean relative LDAC intensity was $97.5 \%$ and $97.4 \%$ for the glasdegib + LDAC and LDAC alone arms, respectively.

All of the patients randomized and treated in both arms reported treatment-emergent AEs during the course of the study. The incidence of AEs was lower over the long term (after 90 days) than the short term (during the first 90 days) in both the glasdegib + LDAC ( $\leq 90$ days, $98.7 \% ;>90$ days, $83.7 \%$ ) and LDAC alone arms ( $\leq 90$ days, $100.0 \% ;>90$ days, $71.4 \%$ ) (Table S3). In the glasdegib + LDAC arm, the most common treatment-emergent AEs over the short term and long term were anemia and diarrhea, respectively. Corresponding AEs in the LDAC alone arm were anemia and anemia/decreased appetite/pneumonia (Table 5). AEs thought to be linked to the inhibition of the Hedgehog signaling pathway in normal tissue occurred in patients receiving glasdegib + LDAC over the short term (alopecia, 4.0\%; dysgeusia, $20.0 \%$; muscle spasms, $14.7 \%$ ) and long-term (alopecia, $9.3 \%$; dysgeusia, $14.0 \%$; muscle spasms, $23.3 \%$ ). The proportion of patients who experienced grade $3 / 4$ AEs was lower over the long term than the short term in both the glasdegib + LDAC ( $\leq 90$ days, $84.0 \% ;>90$ days, $69.8 \%)$ and LDAC alone arms $(\leq 90$ days, $91.7 \%$; $>90$ days, $57.1 \%)$. The severity and rate of AEs were similar in the de novo and secondary AML subgroups (Tables S3 and S4).

Serious AEs were reported in $60 / 75$ patients $(80.0 \%)$ in the glasdegib + LDAC arm and 28/36 patients $(77.8 \%)$ in the LDAC alone arm. The most frequently reported (> 15\% of patients) serious AEs were febrile neutropenia (glasdegib + LDAC, 28.0\%; LDAC alone, 16.7\%) and pneumonia (glasdegib + LDAC, 21.3\%; LDAC alone, 19.4\%). In total,

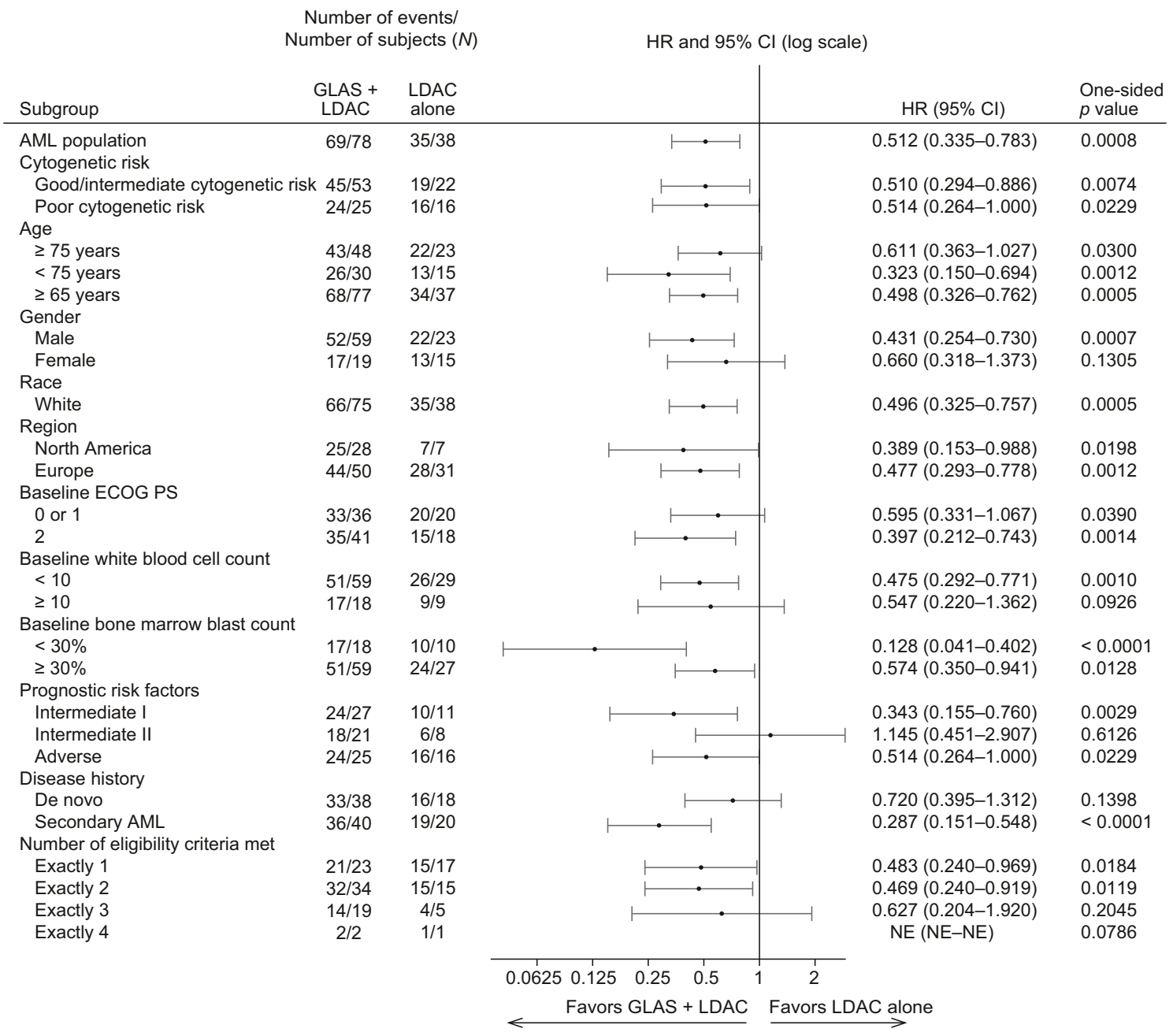

Fig. 2 Forest plot of overall survival for patients with AML (overall population). GLAS, glasdegib; NE, not evaluable 
Table 3 CR in patients with AML

\begin{tabular}{|c|c|c|c|c|c|c|}
\hline & \multicolumn{2}{|l|}{ Overall population } & \multicolumn{2}{|l|}{ de novo AML } & \multicolumn{2}{|l|}{ Secondary AML } \\
\hline & Glasdegib + LDAC & LDAC alone & Glasdegib + LDAC & LDAC alone & Glasdegib + LDAC & LDAC alone \\
\hline & $n=78$ & $n=38$ & $n=38$ & $n=18$ & $n=40$ & $n=20$ \\
\hline Patients with CR, $n(\%)$ & $15(19.2)$ & $1(2.6)$ & $7(18.4)$ & $1(5.6)$ & $8(20.0)^{\mathrm{b}}$ & 0 \\
\hline $95 \% \mathrm{CI}^{\mathrm{a}}$ & $10.5-28.0$ & $0.0-7.7$ & $6.1-30.7$ & $0.0-16.1$ & $7.6-32.4$ & $\mathrm{~N} / \mathrm{A}$ \\
\hline \multicolumn{7}{|l|}{ Duration of remission, days } \\
\hline Median (range) & $302(1-1262)$ & $91(91-91)$ & $175(1-533)$ & $91(91-91)$ & $532(64-1262)$ & 0 \\
\hline \multicolumn{7}{|l|}{ Cytogenetic risk } \\
\hline Good/intermediate risk & $n=49$ & $n=21$ & $n=24$ & $n=14$ & $n=25$ & $n=7$ \\
\hline Patients with CR, $n(\%)$ & $11(22.4)$ & 0 & $5(20.8)$ & 0 & $6(24.0)$ & 0 \\
\hline $95 \%$ exact $\mathrm{CI}^{\mathrm{C}}$ & $11.8-36.6$ & $0.0-16.1$ & $7.1-42.2$ & $0.0-23.2$ & $9.4-45.1$ & $0.0-41.0$ \\
\hline Poor cytogenetic risk & $n=\mathbf{2 9}$ & $n=17$ & $n=14$ & $n=4$ & $n=15$ & $n=13$ \\
\hline Patients with CR, $n(\%)$ & $4(13.8)$ & $1(5.9)$ & $2(14.3)$ & $1(25.0)$ & $2(13.3)$ & 0 \\
\hline $95 \%$ exact $\mathrm{CI}^{\mathrm{c}}$ & $3.9-31.7$ & $0.1-28.7$ & $1.8-42.8$ & $0.6-80.6$ & $1.7-40.5$ & $0.0-24.7$ \\
\hline \multicolumn{7}{|l|}{ Combination vs LDAC } \\
\hline Pearson Chi-square test (unstratified), $p$ value & \multirow{2}{*}{\multicolumn{2}{|c|}{0.0150}} & \multirow{2}{*}{\multicolumn{2}{|c|}{0.1988}} & \multicolumn{2}{|l|}{0.0317} \\
\hline \multicolumn{3}{|c|}{ CMH test stratified by CRF prognosis factor } & & & & \\
\hline Odds ratio $(95 \% \mathrm{CI})$ & \multirow{2}{*}{\multicolumn{2}{|c|}{$4.3762(1.3296-14.4037)$}} & \multirow{2}{*}{\multicolumn{2}{|c|}{$\begin{array}{l}1.8306(0.4931-6.7962) \\
0.2207\end{array}$}} & \multicolumn{2}{|l|}{$4.5370(1.0712-19.2173)$} \\
\hline$p$ value & & & & & 0.0662 & \\
\hline
\end{tabular}

$A M L$, acute myeloid leukemia; $C I$, confidence interval; $C M H$, Cochran-Mantel-Haenszel; $C R$, complete remission; $C R F$, case report form; $L D A C$, lowdose cytarabine; $M D S$, myelodysplastic syndrome; $N / A$, not applicable

${ }^{\text {a } U s i n g ~ n o r m a l ~ a p p r o x i m a t i o n ~}$

${ }^{\mathrm{b}}$ Secondary AML developed from prior MDS $(n=7)$ or from prior chemotherapy/radiotherapy treatment $(n=1)$

${ }^{\mathrm{c}}$ Using exact method based on binomial distribution

$37.3 \%$ of patients who received glasdegib + LDAC and $47.2 \%$ receiving LDAC alone permanently discontinued treatment due to AEs. There were no discontinuations resulting from Hedgehog-inhibitor-class-effect AEs (muscle spasms, ageusia/dysgeusia, alopecia, weight loss or asthenia). The main cause of death in both treatment arms was disease progression.

\section{Biomarkers}

Eighty-three patients had baseline mutational analyses of bone marrow and/or peripheral blood: 41 with de novo AML (glasdegib + LDAC, $n=30$; LDAC alone, $n=11$ ) and 42 with secondary AML (glasdegib + LDAC, $n=28$; LDAC alone, $n=14$ ). For each mutation, only a relatively small number of patients were evaluable. Thus, the effect of baseline mutations on OS was determined in genes with a mutational frequency of $\geq 5$ mutations (Online Resource, Table S5). In the de novo AML subgroup of patients receiving glasdegib + LDAC, there was no statistical $(p>$ 0.05 ) difference in OS for the five genes with a mutational frequency of $>5$ mutations: DNMT3A, combined FLT3 (ITD and TKD), IDH1,IDH2I, and RUNX1. No genes in the LDAC alone arm had $\geq 5$ mutations. For patients in the secondary AML subgroup, $\geq 5$ mutations were reported in three genes in the glasdegib + LDAC arm (DNMT3A, $R U N X 1$, and TET2) and one gene in the LDAC alone arm (TET2). Of the three genes in the glasdegib + LDAC arm,
OS correlated only with mutations in DNMT3A (HR 4.35; 95\% CI 1.40-13.53; $p=0.0056$ ), where patients with secondary AML and mutated DNMT3A had a shorter OS than patients with wild-type DNMT3A; the mutational status of $D N M T 3 A$ did not influence OS in patients with de novo AML. TET2 was the only mutation frequent enough to allow for a comparison between the two treatment arms, and no correlation between OS and mutational status was observed $(p>0.05)$.

\section{Discussion}

This long-term ( $>40$ months) analysis of the BRIGHT 1003 AML study confirms and expands upon previously reported results by demonstrating a statistically significant improvement in survival among patients with AML receiving glasdegib + LDAC versus LDAC alone [18, 19]. The survival benefit of glasdegib was observed across subgroups defined by baseline characteristic. Post-hoc subgroup analyses demonstrated improved OS with glasdegib + LDAC versus LDAC alone in both patients with de novo AML and secondary AML. Furthermore, improvement was consistent across groups stratified by cytogenetic risk, except in patients with de novo AML plus poor-risk cytogenetics where the small sample size precluded meaningful comparisons. 
Table 4 Recovery of ANC, hemoglobin, and platelets, and rates of transfusions in patients with AML (overall population)

\begin{tabular}{|c|c|c|c|c|}
\hline & $\begin{array}{l}\text { Glasdegib + LDAC } \\
n=72\end{array}$ & $\begin{array}{l}\text { LDAC alone } \\
n=32\end{array}$ & $\begin{array}{l}\text { Glasdegib + LDAC } \\
n=72\end{array}$ & $\begin{array}{l}\text { LDAC alone } \\
n=32\end{array}$ \\
\hline ANC & $\geq 1000 / \mu \mathrm{L}$ & & $\geq 500 / \mu \mathrm{L}$ & \\
\hline All patients with recovery, $n(\%)$ & $49(68.1)$ & $20(62.5)$ & $58(80.6)$ & $24(57.1)$ \\
\hline Recovery at $\geq 2$ consecutive visits, $n(\%)$ & $40(55.6)$ & $12(37.5)$ & $47(65.3)$ & $17(53.1)$ \\
\hline Baseline ANC $<$ threshold, $n(\%)^{\mathrm{a}}$ & $22(30.5)$ & $5(15.6)$ & $18(25.0)$ & $1(3.1)$ \\
\hline Median time to first recovery, days (range) & $27(7-114)$ & $13(8-70)$ & $16(3-143)$ & $11(8-119)$ \\
\hline Achieved recovery during cycle $2 / 1, n(\%)^{\mathrm{b}}$ & $30(41.7)$ & $10(31.3)$ & $49(68.1)$ & $22(68.8)$ \\
\hline Hemoglobin & $10 \mathrm{~g} / \mathrm{dL}$ & & $\geq 9 \mathrm{~g} / \mathrm{dL}$ & \\
\hline All patients with recovery, $n(\%)$ & $43(59.7)$ & $18(56.3)$ & $64(88.9)$ & $22(68.8)$ \\
\hline Recovery at $\geq 2$ consecutive visits, $n(\%)$ & $23(31.9)$ & $7(21.9)$ & $44(61.1)$ & $13(40.6)$ \\
\hline Baseline hemoglobin $<$ threshold, $n(\%)^{\mathrm{a}}$ & $19(26.4)$ & $4(12.5)$ & $24(33.3)$ & $2(6.3)$ \\
\hline Median time to first recovery, days (range) & $22(6-129)$ & $33(9-140)$ & $14(4-172)$ & $22(2-85)$ \\
\hline Achieved recovery during cycle $1, n(\%)$ & $31(43.1)$ & $11(34.4)$ & $57(79.2)$ & $20(62.5)$ \\
\hline Platelets & $\geq 100,000 / \mu \mathrm{L}$ & & 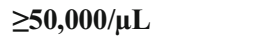 & \\
\hline All patients with recovery, $n(\%)$ & $36(50.0)$ & $7(21.9)$ & $49(68.1)$ & $13(40.6)$ \\
\hline Recovery at $\geq 2$ consecutive visits, $n(\%)$ & $30(41.7)$ & $4(12.5)$ & $38(52.8)$ & $8(25.5)$ \\
\hline Baseline platelets $<$ threshold, $n(\%)^{\mathrm{a}}$ & $20(27.8)$ & $2(6.25)$ & $15(20.8)$ & $3(9.4)$ \\
\hline Median time to first recovery, days (range) & $30(6-171)$ & $26(2-56)$ & $26(4-141)$ & $24(2-119)$ \\
\hline Achieved recovery during cycle $1, n(\%)$ & $24(33.3)$ & $6(18.8)$ & $40(55.6)$ & $11(34.4)$ \\
\hline Transfusion rates & $\begin{array}{l}\text { Glasdegib + LDAC } \\
n=75\end{array}$ & & $\begin{array}{l}\text { LDAC alone } \\
n=36\end{array}$ & \\
\hline \multicolumn{5}{|l|}{ Proportion independent, $n(\%)^{\mathrm{c}}$} \\
\hline No transfusions & $22(29.3)$ & & $2(5.6)$ & \\
\hline $\mathrm{PRBC}$ transfusions & $25(33.3)$ & & $3(8.3)$ & \\
\hline Platelet transfusion & $32(42.7)$ & & $4(11.1)$ & \\
\hline \multicolumn{5}{|l|}{ Exposure-adjusted rate } \\
\hline Any transfusion & 0.0696 & & 0.1555 & \\
\hline PRBC transfusion & 0.0423 & & 0.0789 & \\
\hline Platelet transfusion & 0.0273 & & 0.0766 & \\
\hline
\end{tabular}

$A M L$, acute myeloid leukemia; $A N C$, absolute neutrophil count; $L D A C$, low-dose cytarabine; $P R B C$, packed red blood cell

${ }^{\text {a }}$ Requires measurement at $\geq 2$ consecutive visits

${ }^{\mathrm{b}}$ Cycle 2 for $\mathrm{ANC} \geq 1000 / \mu \mathrm{L}$ and cycle 1 for $\mathrm{ANC} \geq 500 / \mu \mathrm{L}$

${ }^{\mathrm{c}}$ Required no PRBC or platelet transfusions for $\geq 8$ weeks

In the exploratory post-hoc analysis, the survival benefit with glasdegib + LDAC (vs LDAC alone) was most pronounced in patients with secondary AML (HR 0.287; 95\% CI $0.151-0.548 ; p<0.0001)$. Among patients receiving glasdegib + LDAC, observed CR rates were similar whether patients were diagnosed with de novo $(18.4 \%)$ or secondary AML (20.0\%). As previously demonstrated in the overall population in this study, the addition of glasdegib to LDAC significantly improved OS, even among patients who did not achieve CR [23]. Together, these data suggest that patients may benefit from receiving glasdegib in the absence of remission, especially in those with secondary AML.

Secondary AML is biologically distinct from de novo AML. Unique biological features such as subclonal heterogeneity, upregulation of anti-apoptotic proteins, and multidrug resistance make secondary AML treatment challenging [16]. Low blast count AML (20-30\% blasts) is genetically and clinically similar to high-risk MDS, and the response to HMAs and prognosis is comparable between these subgroups [24, 25]. In view of these favorable responses to glasdegib + LDAC, this approach is being investigated in patients with higherrisk MDS [26]. In the primary analysis of the BRIGHT 1003 AML study, a $22.8 \%$ reduction in the risk of death for glasdegib + LDAC relative to LDAC alone was observed in a small subset of patients with MDS [18]. The impact of glasdegib + azacitidine in patients with previously untreated MDS is also currently under evaluation in a single-arm phase II trial (ClinicalTrials. gov identifier: NCT02367456). 
Table 5 Treatment-emergent all-causality AEs occurring in $\geq 20 \%$ of patients (overall population) in any treatment arm during the first 90 days and after 90 days of therapy

\begin{tabular}{|c|c|c|c|c|c|c|}
\hline \multirow[t]{2}{*}{ MedDRA preferred term, $n(\%)^{\mathrm{a}}$} & \multicolumn{3}{|c|}{ Glasdegib + LDAC } & \multicolumn{3}{|c|}{ LDAC alone } \\
\hline & Grade 3-4 & Grade 5 & All AEs & Grade 3-4 & Grade 5 & All AEs \\
\hline During the first 90 days & $n=75$ & & & $n=36$ & & \\
\hline Any AEs & $53(70.7)$ & $12(16.0)$ & $74(98.7)$ & $20(55.6)$ & $13(36.1)$ & $36(100.0)$ \\
\hline Anemia & $31(41.3)$ & 0 & $33(44.0)$ & $13(36.1)$ & 0 & $15(41.7)$ \\
\hline Febrile neutropenia & $23(30.7)$ & 0 & $23(30.7)$ & $8(22.2)$ & 0 & $8(22.2)$ \\
\hline Thrombocytopenia & $23(30.7)$ & 0 & $23(30.7)$ & $8(22.2)$ & 0 & $9(25.0)$ \\
\hline Nausea & $1(1.3)$ & 0 & $22(29.3)$ & $1(2.8)$ & 0 & $4(11.1)$ \\
\hline Fatigue & $7(9.3)$ & 0 & $19(25.3)$ & $2(5.6)$ & 0 & $6(16.7)$ \\
\hline Edema peripheral & 0 & 0 & $17(22.7)$ & $1(2.8)$ & 0 & $7(19.4)$ \\
\hline Constipation & $1(1.3)$ & 0 & $15(20.0)$ & 0 & 0 & $5(13.9)$ \\
\hline Decreased appetite & 0 & 0 & $15(20.0)$ & $1(2.8)$ & 0 & $3(8.3)$ \\
\hline Dysgeusia & 0 & 0 & $15(20.0)$ & 0 & 0 & $1(2.8)$ \\
\hline Pyrexia & $1(1.3)$ & 0 & $15(20.0)$ & $1(2.8)$ & 0 & $8(22.2)$ \\
\hline Vomiting & $2(2.7)$ & 0 & $15(20.0)$ & $1(2.8)$ & 0 & $3(8.3)$ \\
\hline Pneumonia & $8(10.7)$ & $3(4.0)$ & 14 (18.7) & $7(19.4)$ & $1(2.8)$ & $9(25.0)$ \\
\hline Diarrhea & $1(1.3)$ & 0 & $13(17.3)$ & 0 & 0 & $9(25.0)$ \\
\hline Dyspnea & $4(5.3)$ & 0 & $13(17.3)$ & $2(5.6)$ & 0 & $9(25.0)$ \\
\hline After 90 days & $n=43$ & & & $n=14$ & & \\
\hline Any AEs & $22(51.2)$ & $10(23.3)$ & $36(83.7)$ & $6(42.9)$ & $3(21.4)$ & $10(71.4)$ \\
\hline Diarrhea & $3(7.0)$ & 0 & $14(32.6)$ & $1(7.1)$ & 0 & $1(7.1)$ \\
\hline Anemia & $10(23.3)$ & 0 & $13(30.2)$ & $3(21.4)$ & 0 & $3(21.4)$ \\
\hline Decreased appetite & $3(7.0)$ & 0 & $13(30.2)$ & $2(14.3)$ & 0 & $3(21.4)$ \\
\hline Muscle spasms & $4(9.3)$ & 0 & $10(23.3)$ & 0 & 0 & 0 \\
\hline Pyrexia & $1(2.3)$ & 0 & $10(23.3)$ & $1(7.1)$ & 0 & $1(7.1)$ \\
\hline Thrombocytopenia & $9(20.9)$ & 0 & $10(23.3)$ & $2(14.3)$ & 0 & $2(14.3)$ \\
\hline Nausea & $1(2.3)$ & 0 & $9(20.9)$ & 0 & 0 & 0 \\
\hline Neutropenia & $7(16.3)$ & 0 & $9(20.9)$ & $1(7.1)$ & 0 & $1(7.1)$ \\
\hline Pneumonia & $3(7.0)$ & $3(7.0)$ & 8 (18.6) & $1(7.1)$ & $2(14.3)$ & 3 (21.4) \\
\hline
\end{tabular}

$A E$, adverse event; $L D A C$, low-dose cytarabine; MedDRA, Medical Dictionary for Regulatory Activities

${ }^{\text {a }}$ Patients are counted only once per preferred term in each row. Each count is based on the maximum grade of events

Preliminary signs of clinical efficacy were evident across patients with diverse mutational profiles; however, small patient numbers prevent firm conclusions from being drawn based on mutational status for some genes in this analysis. In the de novo AML subgroup, there was no statistical difference in OS according to the mutational status of DNMT3A, combined FLT3, IDH1, $I D H 2$, and RUNX1 genes in the glasdegib + LDAC arm. For patients in the secondary AML subgroup, there was no statistical difference in OS according to mutational status of RUNX1 and TET2 genes in the glasdegib + LDAC arm; however, there was a negative correlation with mutations in DNMT3A $(p=0.0056)$ compared with patients with wild-type DNMT3A. DNMT3A mutations were associated with a short OS in both the glasdegib + LDAC and LDAC alone arms
(3.1 and 1.9 months, respectively). DNMT3A is a DNA methyltransferase that catalyzes the transfer of a methyl group onto the 5'-position of cytosine of $\mathrm{CpG}$ dinucleotides. Mutations in DNMT3A are thought to play a pivotal role in the initiation of clonal hematopoiesis and provide a fertile ground for AML transformation. The prognostic implications of DNMT3A mutations are inconclusive, with a number of studies suggesting that mutations of DNMT3A confer a poor prognosis [27]. In this study, mutational status of DNMT3A correlated with OS during glasdegib + LDAC treatment in patients with secondary AML, but not in patients with de novo AML.

Long-term follow-up confirmed that treatment with glasdegib + LDAC was associated with an acceptable safety profile in patients with AML, with little additional toxicity seen with the combination of glasdegib + LDAC versus 
LDAC alone. No patients discontinued treatment because of AEs associated with inhibition of the Hedgehog signaling pathway in normal tissues (e.g., muscle spasms, ageusia/ dysgeusia, alopecia, weight loss, and asthenia). The smoothened antagonists vismodegib and sonidegib are approved for the treatment of advanced basal cell carcinoma; however, their efficacy has yet to be demonstrated in the treatment of AML [28-30]. Although the on-target profile of glasdegib is consistent with both agents, the rate and severity of AEs are lower with glasdegib treatment. The most commonly occurring AEs with vismodegib and sonidegib included alopecia, dysgeusia, and muscle spasms. The differences between the safety profiles may in part be explained by the shorter elimination halflife of glasdegib (sonidegib, 28 days; vismodegib, 4 days; glasdegib, $17.4 \mathrm{~h}$ ) [28, 29, 31].

As the Hedgehog signaling pathway is not essential for adult hematopoietic stem cell function, targeting leukemic stem cells with glasdegib may allow a reduction in tumor burden while maintaining normal hematopoiesis [32, 33]. Patients receiving glasdegib + LDAC had improved celllineage recovery data, suggesting that the treatment combination provides clinical benefit by reducing the risk of cytopenias in this patient population. More patients receiving glasdegib + LDAC were transfusion-independent and, when exposure-adjusted, LDAC alone patients required transfusions twice as often. These results were consistent in both patients with de novo and secondary AML.

Until recently, the general therapeutic strategy for patients with AML who are ineligible for intensive chemotherapy was treatment with LDAC or HMAs. A meta-analysis including patients unable to receive intensive chemotherapy, because of comorbidities or older age ( $\geq 75$ years), demonstrated that the median OS for patients receiving treatment with azacitidine, decitabine, or LDAC was 6.3 months [34]. The recent US Food and Drug Administration approvals of glasdegib and venetoclax in combination with standard-of-care therapies has provided new therapeutic options for this patient population $[17,18,35]$. In this study, the combination of glasdegib + LDAC was shown to be most beneficial in patients with secondary AML. Although comparison between trials should be considered with caution due to potential methodologic and other differences, the median OS for patients with secondary AML receiving glasdegib + LDAC compared favorably with previously reported outcomes in patients receiving enasidenib (8.8 months), decitabine (7.1 months), and venetoclax + LDAC (4.0 months) $[13,35,36]$. In addition, the median OS with glasdegib + LDAC was comparable to that in patients with secondary or therapyrelated AML who were eligible for intensive chemotherapy and who received treatment with CPX-351 (9.6 months) [10]. Considering the specified criteria to select patients who were ineligible for intensive chemotherapy in our study, with a median age nearly a decade older than in the CPX-351 study, and more than half of patients with an ECOG PS of 2 (compared with $<10 \%$ in the CPX-351 study), as well as significant comorbidities (e.g., cardiac disease in $>50 \%$ ), this analysis suggests that glasdegib + LDAC is a valuable alternative for these patients.

In conclusion, long-term analysis of the BRIGHT AML 1003 study continued to show superior OS with glasdegib + LDAC (vs LDAC alone) in patients with AML who were ineligible for intensive chemotherapy. The clinical benefit with glasdegib + LDAC was most pronounced in patients with secondary AML, with a statistically significant and clinically meaningful improvement in OS compared with LDAC alone. The combination of glasdegib + LDAC represents a promising treatment strategy for patients with secondary AML who are ineligible for intensive chemotherapy. Glasdegib is currently in phase III clinical development (BRIGHT AML 1019; ClinicalTrials.gov identifier: NCT03416179) for patients with AML in combination with azacitidine or $7+3$ intensive chemotherapy.

Supplementary Information The online version contains supplementary material available at https://doi.org/10.1007/s00277-021-04465-4.

Acknowledgements The authors would like to thank all patients who participated in the trial and medical staff of participating centers. Medical writing support was provided by Gary Dever, PhD, and Gemma Shay, PhD, of Engage Scientific Solutions, and was funded by Pfizer.

Author contribution Conception or design of the study: Jorge E. Cortes, Caroline J. Hoang, Thomas O'Brien, Weidong Wendy Ma, Mirjana Zeremski, Ashleigh O'Connell, and Geoffrey Chan.

Acquisition of data: Michael Heuser, B. Douglas Smith, Walter Fiedler, Mikkael A. Sekeres, Pau Montesinos, Brian Leber, Akil Merchant, Cristina Papayannidis, José A. Pérez-Simón, and Jorge E. Cortes.

All authors contributed equally to the analysis and interpretation of data. All authors contributed equally to the preparation and final approval of the manuscript.

Funding Open Access funding enabled and organized by Projekt DEAL. This study was funded by Pfizer.

Data availability Upon request, and subject to certain criteria, conditions, and exceptions (see https://www.pfizer.com/science/clinical-trials/trialdata-and-results for more information), Pfizer will provide access to individual de-identified participant data from Pfizer-sponsored global interventional clinical studies conducted for medicines, vaccines, and medical devices (1) for indications that have been approved in the USA and/or EU, or (2) in programs that have been terminated (i.e., development for all indications has been discontinued). Pfizer will also consider requests for the protocol, data dictionary, and statistical analysis plan. Data may be requested from Pfizer trials 24 months after study completion. The de- 
identified participant data will be made available to researchers whose proposals meet the research criteria and other conditions, and for which an exception does not apply, via a secure portal. To gain access, data requestors must enter into a data access agreement with Pfizer.

Code availability Not applicable.

\section{Declarations}

Ethics approval The study was conducted in accordance with the Declaration of Helsinki. The protocol was approved by institutional review boards at each study site.

Consent to participate All patients provided written informed consent before study procedures began.

Consent for publication This manuscript has been approved by all authors.

Conflicts of interest/Competing interests Michael Heuser received research funding and honoraria from Pfizer, prIME Oncology, and Jazz Pharmaceuticals; served on advisory boards for AbbVie, Bayer Pharma AG, Daiichi Sankyo, Novartis, Pfizer, and Jazz Pharmaceuticals; and received research funding to his institute from Astellas, Bayer Pharma AG, BerGenBio, Daiichi Sankyo, Karyopharm, Novartis, Pfizer, and Roche. B. Douglas Smith served on an advisory board and was a consultant for Celgene, Jazz Pharmaceuticals, Novartis, and Pfizer. Walter Fiedler has participated in advisory boards for Amgen, Pfizer, Novartis, Jazz Pharmaceuticals, and ARIAD/Incyte; received research funding from Amgen; received support for meeting attendance from Amgen, Jazz Pharmaceuticals, Daiichi Sankyo Oncology, and Servier; and has received support for medical writing from Amgen, Pfizer, and AbbVie. Mikkael A. Sekeres is an advisory board member for Celgene. Pau Montesinos served on an advisory board for Celgene, Jazz Pharmaceuticals, Janssen, and Novartis; and has received research funding from Pfizer and Celgene. Brian Leber has received honoraria and served on advisory boards for Novartis, Pfizer, Celgene, AbbVie, Astellas, Jazz Pharmaceuticals, and Alexion; and was a consultant for Novartis and Pfizer. Akil Merchant is an advisory board member for Agios, Pfizer, and Takeda; and has received research funding from Pfizer. Cristina Papayannidis has received honoraria from Pfizer, Novartis, Incyte, and Amgen. José A. Pérez-Simón has received honoraria and or/research funding from Pfizer, Jazz Pharmaceuticals, Takeda, Amgen, Janssen, and Novartis. Caroline J. Hoang, Thomas O'Brien, Weidong Wendy Ma, Mirjana Zeremski, Ashleigh O'Connell, and Geoffrey Chan are all employees and shareholders of Pfizer. Jorge E. Cortes received research funding to his institution and consulting honoraria from Pfizer, Novartis, Astellas, Daiichi Sankyo, Jazz Pharmaceuticals, Takeda, and Celgene.

Open Access This article is licensed under a Creative Commons Attribution 4.0 International License, which permits use, sharing, adaptation, distribution and reproduction in any medium or format, as long as you give appropriate credit to the original author(s) and the source, provide a link to the Creative Commons licence, and indicate if changes were made. The images or other third party material in this article are included in the article's Creative Commons licence, unless indicated otherwise in a credit line to the material. If material is not included in the article's Creative Commons licence and your intended use is not permitted by statutory regulation or exceeds the permitted use, you will need to obtain permission directly from the copyright holder. To view a copy of this licence, visit http://creativecommons.org/licenses/by/4.0/.

\section{References}

1. Harris NL, Jaffe ES, Diebold J, Flandrin G, Muller-Hermelink HK, Vardiman J, Lister TA, Bloomfield CD (1999) World Health Organization classification of neoplastic diseases of the hematopoietic and lymphoid tissues: report of the Clinical Advisory Committee meeting-Airlie House, Virginia, November 1997. J Clin Oncol 17(12):3835-3849. https://doi.org/10.1200/JCO.1999. 17.12.3835

2. Schoch C, Kern W, Schnittger S, Buchner T, Hiddemann W, Haferlach T (2004) The influence of age on prognosis of de novo acute myeloid leukemia differs according to cytogenetic subgroups. Haematologica 89(9):1082-1090

3. Grimwade D, Hills RK, Moorman AV, Walker H, Chatters S, Goldstone AH, Wheatley K, Harrison CJ, Burnett AK, National Cancer Research Institute Adult Leukaemia Working Group (2010) Refinement of cytogenetic classification in acute myeloid leukemia: determination of prognostic significance of rare recurring chromosomal abnormalities among 5876 younger adult patients treated in the United Kingdom Medical Research Council trials. Blood 116(3):354-365. https://doi.org/10.1182/blood-2009-11254441

4. Grimwade D, Hills RK (2009) Independent prognostic factors for AML outcome. Hematology Am Soc Hematol Educ Program (1): 385-395. https://doi.org/10.1182/asheducation-2009.1.385

5. Ostgard LS, Kjeldsen E, Holm MS, Brown Pde N, Pedersen BB, Bendix K, Johansen P, Kristensen JS, Norgaard JM (2010) Reasons for treating secondary AML as de novo AML. Eur J Haematol 85(3):217-226. https://doi.org/10.1111/j.1600-0609.2010.01464.x

6. Kayser S, Dohner K, Krauter J, Kohne CH, Horst HA, Held G, von Lilienfeld-Toal M, Wilhelm S, Kundgen A, Gotze K, Rummel M, Nachbaur D, Schlegelberger B, Gohring G, Spath D, Morlok C, Zucknick M, Ganser A, Dohner H, Schlenk RF, German-Austrian AMLSG (2011) The impact of therapy-related acute myeloid leukemia (AML) on outcome in 2853 adult patients with newly diagnosed AML. Blood 117(7):2137-2145. https://doi.org/10.1182/ blood-2010-08-301713

7. Hulegardh E, Nilsson C, Lazarevic V, Garelius H, Antunovic P, Rangert Derolf A, Mollgard L, Uggla B, Wennstrom L, Wahlin A, Hoglund M, Juliusson G, Stockelberg D, Lehmann S (2015) Characterization and prognostic features of secondary acute myeloid leukemia in a population-based setting: a report from the Swedish Acute Leukemia Registry. Am J Hematol 90(3):208214. https://doi.org/10.1002/ajh.23908

8. Juliusson G, Antunovic P, Derolf A, Lehmann S, Mollgard L, Stockelberg D, Tidefelt U, Wahlin A, Hoglund M (2009) Age and acute myeloid leukemia: real world data on decision to treat and outcomes from the Swedish Acute Leukemia Registry. Blood 113(18):4179-4187. https://doi.org/10.1182/blood-2008-07172007

9. Schoch C, Kern W, Schnittger S, Hiddemann W, Haferlach T (2004) Karyotype is an independent prognostic parameter in therapy-related acute myeloid leukemia (t-AML): an analysis of 93 patients with t-AML in comparison to 1091 patients with de novo AML. Leukemia 18(1):120-125. https://doi.org/10.1038/sj. leu.2403187

10. Lancet JE, Uy GL, Cortes JE, Newell LF, Lin TL, Ritchie EK, Stuart RK, Strickland SA, Hogge D, Solomon SR, Stone RM, Bixby DL, Kolitz JE, Schiller GJ, Wieduwilt MJ, Ryan DH, Hoering A, Banerjee K, Chiarella M, Louie AC, Medeiros BC (2018) CPX-351 (cytarabine and daunorubicin) liposome for injection versus conventional cytarabine plus daunorubicin in older patients with newly diagnosed secondary acute myeloid leukemia. J Clin Oncol 36(26):2684-2692. https://doi.org/10.1200/JCO.2017. 77.6112 
11. Klepin HD, Rao AV, Pardee TS (2014) Acute myeloid leukemia and myelodysplastic syndromes in older adults. J Clin Oncol 32(24):2541-2552. https://doi.org/10.1200/JCO.2014.55.1564

12. Heuser M, Ofran Y, Boissel N, Brunet Mauri S, Craddock C, Janssen J, Wierzbowska A, Buske C, ESMO Guidelines Committee (2020) Acute myeloid leukaemia in adult patients: ESMO Clinical Practice Guidelines for diagnosis, treatment and follow-up. Ann Oncol 31:697-712. https://doi.org/10.1016/j. annonc.2020.02.018

13. Kantarjian HM, Thomas XG, Dmoszynska A, Wierzbowska A, Mazur G, Mayer J, Gau JP, Chou WC, Buckstein R, Cermak J, Kuo CY, Oriol A, Ravandi F, Faderl S, Delaunay J, Lysak D, Minden M, Arthur C (2012) Multicenter, randomized, open-label, phase III trial of decitabine versus patient choice, with physician advice, of either supportive care or low-dose cytarabine for the treatment of older patients with newly diagnosed acute myeloid leukemia. J Clin Oncol 30(21):2670-2677. https://doi.org/10. 1200/JCO.2011.38.9429

14. Dohner H, Lubbert M, Fiedler W, Fouillard L, Haaland A, Brandwein JM, Lepretre S, Reman O, Turlure P, Ottmann OG, Muller-Tidow C, Kramer A, Raffoux E, Dohner K, Schlenk RF, Voss F, Taube T, Fritsch H, Maertens J (2014) Randomized, phase 2 trial of low-dose cytarabine with or without volasertib in AML patients not suitable for induction therapy. Blood 124(9):14261433. https://doi.org/10.1182/blood-2014-03-560557

15. Dombret H, Seymour JF, Butrym A, Wierzbowska A, Selleslag D, Jang JH, Kumar R, Cavenagh J, Schuh AC, Candoni A, Recher C, Sandhu I, Bernal del Castillo T, Al-Ali HK, Martinelli G, Falantes J, Noppeney R, Stone RM, Minden MD, McIntyre H, Songer S, Lucy LM, Beach CL, Dohner H (2015) International phase 3 study of azacitidine vs conventional care regimens in older patients with newly diagnosed AML with $>30 \%$ blasts. Blood 126(3):291-299. https://doi.org/10.1182/blood-2015-01-621664

16. Kuykendall A, Duployez N, Boissel N, Lancet JE, Welch JS (2018) Acute myeloid leukemia: the good, the bad, and the ugly. Am Soc Clin Oncol Educ Book 38:555-573. https://doi.org/10.1200/ EDBK 199519

17. Bohl SR, Bullinger L, Rucker FG (2019) New targeted agents in acute myeloid leukemia: new hope on the rise. Int J Mol Sci 20(8): E1983. https://doi.org/10.3390/ijms20081983

18. Cortes JE, Heidel FH, Hellmann A, Fiedler W, Smith BD, Robak T, Montesinos P, Pollyea DA, DesJardins P, Ottmann O, Ma WW, Shaik MN, Laird AD, Zeremski M, O'Connell A, Chan G, Heuser M (2019) Randomized comparison of low dose cytarabine with or without glasdegib in patients with newly diagnosed acute myeloid leukemia or high-risk myelodysplastic syndrome. Leukemia 33(2): 379-389. https://doi.org/10.1038/s41375-018-0312-9

19. Pfizer Inc (2018) DAURISMO® (glasdegib) prescribing information. Pfizer Labs. https://www.accessdata.fda.gov/drugsatfda_docs/ label/2018/210656s0001bl.pdf. Accessed 14 July 2019

20. Pfizer Europe MA EEIG (2020) Summary of product characteristics: DAURISMO ${ }^{\mathrm{TM}}$ (glasdegib). https://www.ema.europa.eu/en/ documents/product-information/daurismo-epar-productinformation_en.pdf. Accessed 14 August 2020

21. Cheson BD, Bennett JM, Kopecky KJ, Buchner T, Willman CL, Estey EH, Schiffer CA, Doehner H, Tallman MS, Lister TA, LoCoco F, Willemze R, Biondi A, Hiddemann W, Larson RA, Lowenberg B, Sanz MA, Head DR, Ohno R, Bloomfield CD, International Working Group for Diagnosis, Standardization of Response Criteria Treatment Outcomes, Reporting Standards for Therapeutic Trials in Acute Myeloid Leukemia (2003) Revised recommendations of the International Working Group for Diagnosis, Standardization of Response Criteria, Treatment Outcomes, and Reporting Standards for Therapeutic Trials in Acute Myeloid Leukemia. J Clin Oncol 21(24):4642-4649. https://doi.org/10.1200/JCO.2003.04.036
22. Döhner H, Estey EH, Amadori S, Appelbaum FR, Büchner T, Burnett AK, Dombret H, Fenaux P, Grimwade D, Larson RA, Lo-Coco F, Naoe T, Niederwieser D, Ossenkoppele GJ, Sanz MA, Sierra Jorge, Tallman MS, Löwenberg B, Bloomfield CD (2010) Diagnosis and management of acute myeloid leukemia in adults: recommendations from an international expert panel, on behalf of the European LeukemiaNet. Blood 115:453-474. https://doi.org/10.1182/blood-2009-07-235358

23. Cortes JE, Heidel FH, Fiedler W, Smith BD, Robak T, Montesinos P, Candoni A, Leber B, Sekeres MA, Pollyea DA, Ferdinand R, Ma WW, O'Brien T, O'Connell A, Chan G, Heuser M (2020) Survival outcomes and clinical benefit in patients with acute myeloid leukemia treated with glasdegib and low-dose cytarabine according to response to therapy. J Hematol Oncol 13(1):92. https://doi.org/10. 1186/s13045-020-00929-8

24. DiNardo CD, Garcia-Manero G, Pierce S, Nazha A, Bueso-Ramos C, Jabbour E, Ravandi F, Cortes J, Kantarjian H (2016) Interactions and relevance of blast percentage and treatment strategy among younger and older patients with acute myeloid leukemia (AML) and myelodysplastic syndrome (MDS). Am J Hematol 91(2):227232. https://doi.org/10.1002/ajh.24252

25. Fenaux P, Mufti GJ, Hellstrom-Lindberg E, Santini V, Gattermann N, Germing U, Sanz G, List AF, Gore S, Seymour JF, Dombret H, Backstrom J, Zimmerman L, McKenzie D, Beach CL, Silverman LR (2010) Azacitidine prolongs overall survival compared with conventional care regimens in elderly patients with low bone marrow blast count acute myeloid leukemia. J Clin Oncol 28(4):562569. https://doi.org/10.1200/JCO.2009.23.8329

26. Walter MJ, Shen D, Ding L, Shao J, Koboldt DC, Chen K, Larson DE, McLellan MD, Dooling D, Abbott R, Fulton R, Magrini V, Schmidt H, Kalicki-Veizer J, O'Laughlin M, Fan X, Grillot M, Witowski S, Heath S, Frater JL, Eades W, Tomasson M, Westervelt P, DiPersio JF, Link DC, Mardis ER, Ley TJ, Wilson RK, Graubert TA (2012) Clonal architecture of secondary acute myeloid leukemia. N Engl J Med 366(12):1090-1098. https://doi. org/10.1056/NEJMoa1106968

27. Yang L, Rau R, Goodell MA (2015) DNMT3A in haematological malignancies. Nat Rev Cancer 15(3):152-165. https://doi.org/10. $1038 /$ nrc3895

28. Axelson M, Liu K, Jiang X, He K, Wang J, Zhao H, Kufrin D, Palmby T, Dong Z, Russell AM, Miksinski S, Keegan P, Pazdur R (2013) U.S. Food and Drug Administration approval: vismodegib for recurrent, locally advanced, or metastatic basal cell carcinoma. Clin Cancer Res 19(9):2289-2293. https://doi.org/10.1158/10780432.CCR-12-1956

29. Casey D, Demko S, Shord S, Zhao H, Chen H, He K, Putman A, Helms W, Keegan P, Pazdur R (2017) FDA approval summary: sonidegib for locally advanced basal cell carcinoma. Clin Cancer Res 23(10):2377-2381. https://doi.org/10.1158/1078-0432.CCR16-2051

30. Bixby D, Noppeney R, Lin TL, Cortes J, Krauter J, Yee K, Medeiros BC, Kramer A, Assouline S, Fiedler W, Dimier N, Simmons BP, Riehl T, Colburn D (2019) Safety and efficacy of vismodegib in relapsed/refractory acute myeloid leukaemia: results of a phase Ib trial. Br J Haematol 185(3):595-598. https://doi.org/ 10.1111/bjh.15571

31. Norsworthy KJ, By K, Subramaniam S, Zhuang L, Del Valle PL, Przepiorka D, Shen YL, Sheth CM, Liu C, Leong R, Goldberg KB, Farrell AT, Pazdur R (2019) FDA approval summary: glasdegib for newly diagnosed acute myeloid leukemia. Clin Cancer Res 25(20): 6021-6025. https://doi.org/10.1158/1078-0432.CCR-19-0365

32. Gao J, Graves S, Koch U, Liu S, Jankovic V, Buonamici S, El Andaloussi A, Nimer SD, Kee BL, Taichman R, Radtke F, Aifantis I (2009) Hedgehog signaling is dispensable for adult hematopoietic stem cell function. Cell Stem Cell 4(6):548-558. https://doi.org/10.1016/j.stem.2009.03.015 
33. Fukushima N, Minami Y, Kakiuchi S, Kuwatsuka Y, Hayakawa F, Jamieson C, Kiyoi H, Naoe T (2016) Small-molecule Hedgehog inhibitor attenuates the leukemia-initiation potential of acute myeloid leukemia cells. Cancer Sci 107(10):1422-1429. https://doi.org/ 10.1111/cas.13019

34. Stone A, Zukerman T, Flaishon L, Yakar RB, Rowe JM (2019) Efficacy outcomes in the treatment of older or medically unfit patients with acute myeloid leukaemia: a systematic review and metaanalysis. Leuk Res 82:36-42. https://doi.org/10.1016/j.leukres. 2019.05.007

35. DiNardo CD, Pratz K, Pullarkat V, Jonas BA, Arellano M, Becker PS, Frankfurt O, Konopleva M, Wei AH, Kantarjian HM, Xu T, Hong WJ, Chyla B, Potluri J, Pollyea DA, Letai A (2019) Venetoclax combined with decitabine or azacitidine in treatment- naive, elderly patients with acute myeloid leukemia. Blood 133(1): 7-17. https://doi.org/10.1182/blood-2018-08-868752

36. Wei AH, Strickland SA Jr, Hou JZ, Fiedler W, Lin TL, Walter RB, Enjeti A, Tiong IS, Savona M, Lee S, Chyla B, Popovic R, Salem AH, Agarwal S, Xu T, Fakouhi KM, Humerickhouse R, Hong WJ, Hayslip J, Roboz GJ (2019) Venetoclax combined with low-dose cytarabine for previously untreated patients with acute myeloid leukemia: results from a phase Ib/II study. J Clin Oncol 37(15):12771284. https://doi.org/10.1200/JCO.18.01600

Publisher's note Springer Nature remains neutral with regard to jurisdictional claims in published maps and institutional affiliations. 